\title{
Remote Home Monitoring Based on Mobile System
}

\section{Authors: Naqaa Luqmqn Mohammed"; Mohaned L. Ahmed'; Shaima M. Mohamed Najeeb ${ }^{3}$}

\author{
Affiliation: Computer Dep./ Engineering technical college /Northen Technical \\ University /Mosul_Iraq ${ }^{123}$
}

E-mail:naqaa_alhamo@ntu.edu.iq ${ }^{1}$; email ${ }^{2}:$ shaimamiqdad@ntu.edu.iq ${ }^{2}$

DOI: 10.26821/IJSHRE.9.9.2021.9903

\begin{abstract}
:
In recent decades, a smart home has 43, controlling and monitoring the users' home. The administrator and users can manage and control systems' code locally (LAN) or remotely (Internet). The second part is the hardware interface module, which provides a suitable driver and interface for the home sensors. This system is scalable system so that a single server can manage more than one hardware interface modules when it is under Wi-Fi coverage.

Keywords: Smart Home, Wi-Fi, remote controlled, home automation, Android smartphone, Arduino.
\end{abstract}

\section{Introduction:}

Home Monitoring System represents a fantastic great opportunity in establishing new area in engineering, architecture and computing. This system is becoming popular in our days and fast entering in the market. However, end users, especially the disabled and the elderly due to their complexity and cost, do not always accept these systems. Due to the advancement of wireless technology, many different communications have been introduced such as GSM, WIFI, ZIGBEE, and Bluetooth. Each connection has its own unique specifications and applications. It is among the four common wireless connections, WIFI was implemented in our paper [1].

The paper aims to design an advanced home automation system by using the regular web server and Wi-Fi technology. Devices can be switched ON/OFF and sensors can be read by using a PC which under Wi-Fi coverage. Automation is the most spelled term in electronic [2].

Concert the advantages of $\mathrm{Wi}-\mathrm{Fi}$, an advanced automation system has been developed to control devices in the home. Wi-Fi (Short for Wireless Fidelity) is a wireless technology that uses radio frequency to transmit data through the air. Wi-Fi has initial speeds from $1 \mathrm{Mbps}$ to $2 \mathrm{Mbps}$. Wi-Fi transmits data in the $2.4 \mathrm{GHz}$ frequency band. In this paper the automation controller is Arduino UNO. The data which sent from the PC, it will be received via $\mathrm{Wi}-\mathrm{Fi}$ by the $\mathrm{Wi}-\mathrm{Fi}$ module which connected to the Arduino UNO. The Arduino UNO reads the data and decides for switching the electrical devices which connected to it through Relays [3].

Naqaa Luqman Mohammed; Mohaned L. Ahmed; Shaima M. Mohamed Najeeb, Volume 9 Issue 9, pp 8-11 September 2021 
Volume 9 Issue 9 September 2021

\section{OVERVIEW OF THE TECHNOLOGY}

USED

\subsection{Setting up the circuit: -}

The circuit of home automation and security system can be summary as: PIR sensor, Arduino UNO (or other), esp8266-01, and a power supply, which represent the basic circuit for this paper, we can also add many different sensors and other components to the circuit such as a weight sensor to monitor the amount of food in the bowl or bulbs that will change according to different parameters [4].

2.1.1 ESP8266 : is a low-cost Wi-Fi microchip, with a full TCP/IP stack and microcontroller capability . This small unit allows microcontrollers to connect to a Wi-Fi network and make simple TCP/IP connections using Hayes-style commands. The pinout is as follows for the common ESP-01 unit (see figure 1):

1. VCC, Voltage $(+3.3 \mathrm{~V}$; can handle up to $3.6 \mathrm{~V})$.

2. GND, Ground (0 V).

3. RX, Receive data bit X.

4. TX, X bit data Transmission.

5. CH_PD, Chip power-down.

6. RST, Reset.

7. GPIO 0, General-purpose input/output No. 0.

8. GPIO 2, General-purpose input/output No. 2.

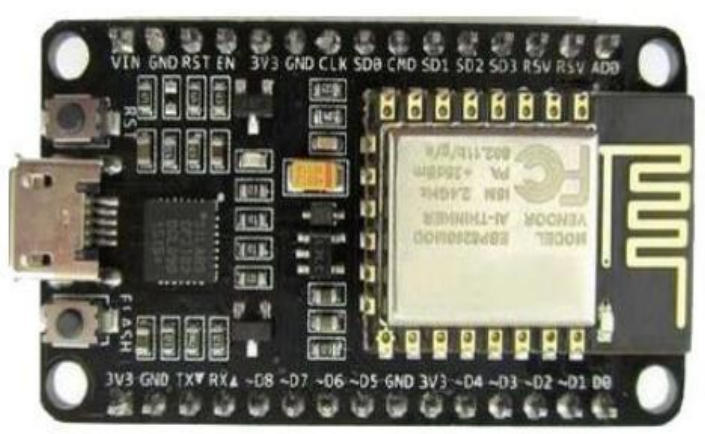

Fig 1. ESP2066

2.1.2 LDR: known LDR resistor-based light also known as optical resistor, or photoelectric cell, or optical connector. It is one type of resistor that changes depending on the amount of light which falls on the its surface. When the light falls on the resistor, the resistance changes. These resistors are often used in many circuits where they are required to sense the presence of light intensity (see figure 2) [5].

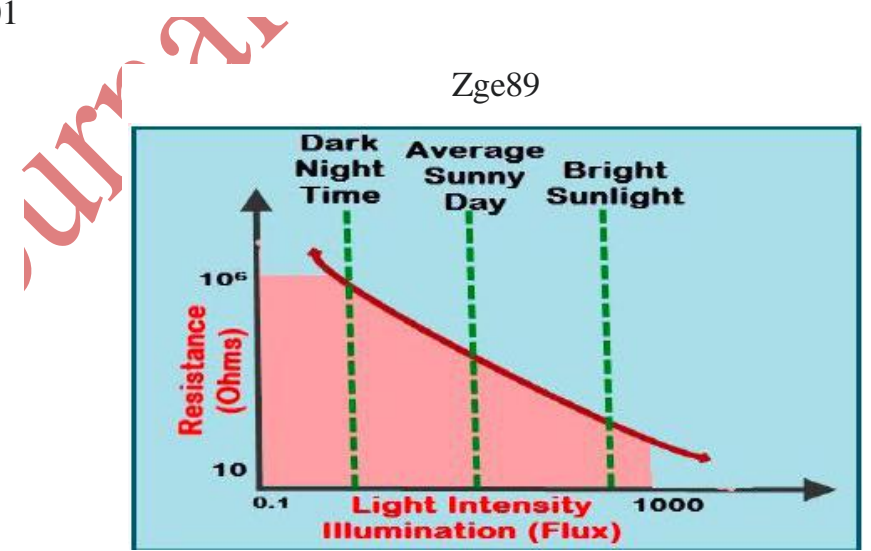

Fig 2. Light Intensity vs LDR Resistance

The connection of LDR is shown in figure 3, when the sensor is sense the light, it will give the instruction to lamp to become off and when the sensor senses there is no light, it will give instruction to lamp to become on. 


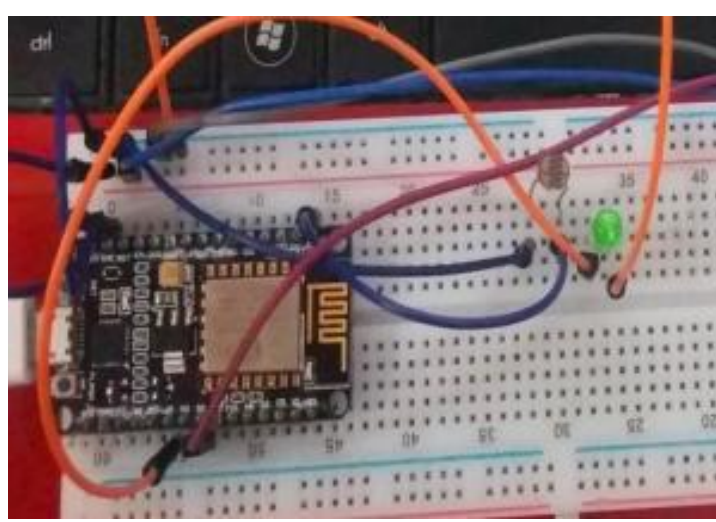

Fig 3. LDR connection

2.1.3 HD11: connect the HD11 sensor to ESP2066 for sensing the degree of tempture [6], when read the degree of tempture is high then give the instruction to fan for running as shown in figure 4:

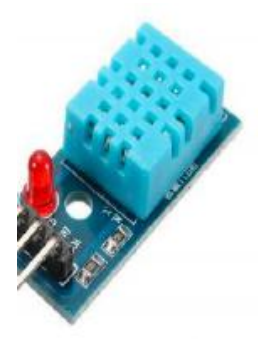

(a)

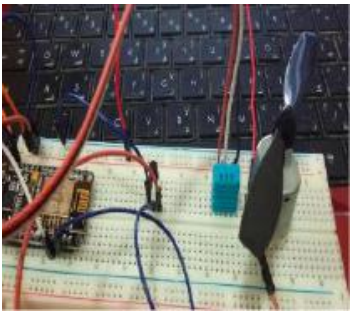

(b)
Fig 4. HD11 connection with fan

\subsection{Circuit connection using android mobile}

In this paper, we connect the three lamps to an Arduino relay circuit to control them by the ESP2066 board which indicates the possibility of WIFI and configure the node-MCU boar as a HTTP server to perform the control of various home appliances such as (heater, lamp, fan, etc.) [7], Figure (5) is the circuit diagram and Figure (6) shows the physical circuit connection.

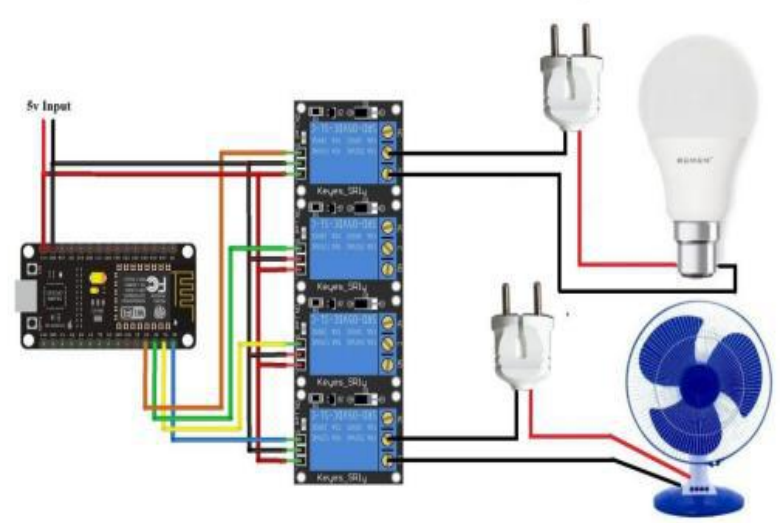

Fig 5. Circuit diagram of lamps connection

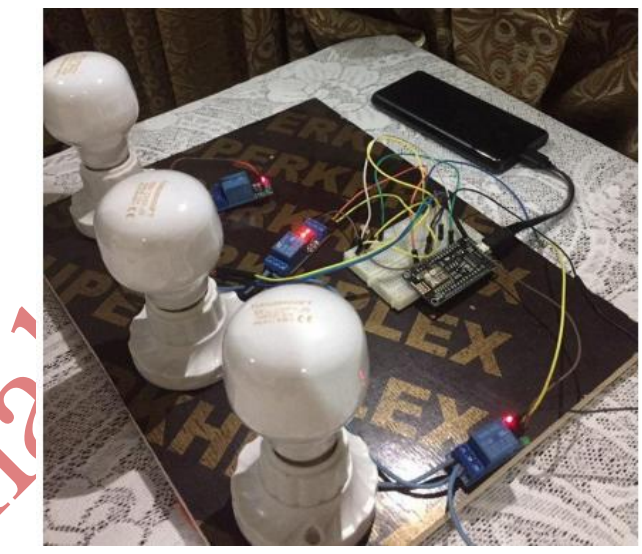

Fig 6. Three lamps with board circuit connection

\section{Software}

In this paper $\mathrm{C}$ language is used to program Arduino app. and MIT is used to program Android phone. The app. consists of main functions like lighting control, fan control, temperature control, etc. The main screen contains a list of a functions that the user can choose one from it. After selecting the function, he will be able to see the current state of a particular device. If the user is so desires, he will cut or deactivate the intended device. The system is also programmed to automatically turn on / off the lights during the late night hours and also turn on or off the air conditioner when the room temperature is too high or too low [8]. 
Volume 9 Issue 9 September 2021

the design face of Android application is shown in figure 7:

\section{HOME AUTOMATION}

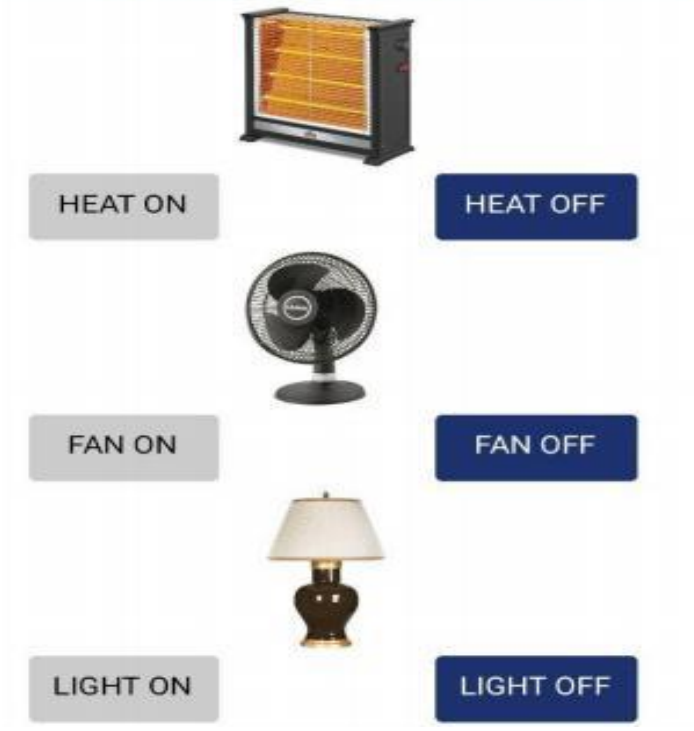

Figure 7. Android application design

\section{Conclusion}

IOT facilitates many benefits to society and from our paper we can provide and demonstrate the strength of IOT that is able to contribute to services for the purpose of building a large number Of applications and assistance in implementing them on the public platform. This design has been successfully tested and provides a moderate, flexible and cost-effective method of sensing, monitoring and controlling in the field of domestic and industry standards to implement IOT. On a final note, we conclude that the Internet of Things is becoming global in every aspect. This paper will be very useful in our normal daily life and will bring a lot of innovation required in the rapidly changing world of technology as well as where people prefer to control things with smart phones that will make their routine life easier.

\section{Reference:}

[1]. Ajinkya Korane, Sanket Salunke "HOME appliances control system based on android smartphone", International journal of Advance Research in Science and Engineering. vol no. 4 ,Issue 07,July 2015.

[2]. Dr. Thida Aung,"Arduino Based Home Lighting Control by Android Phone", August 2019 International Journal of Trend in Scientific Research and Development (IJTSRD) Volume 3 Issue 5, Available Online: www.ijtsrd.com eISSN: $2456-6470$

[3]. Rajeev Piyare1 and Seong Ro Lee1," Smart Home-Control and Monitoring System Using Smart Phone", ASTL Vol. 24, pp. 83 - 86. , ICCA 2013.

[4].Aru O E, Ihekweaba G and Opara F K, 2013,'Design Exploration of a Microcontroller Based RF Remote Control 13amps Wall Socket", IOSR-JCE, 11(1), 56-60.

[5]. Asif O, Hossain B, Hasan M, Rahman T and Chowdhury M," Fire Detectors Review and Design of an Automated, Quick Responsive FireAlarm”. Int J. Communications, Network and System Sciences, 2014, 7, 386-395 Published Online September 2014 in SciRes. h

[6]. Mohammed N L, Asis. M SH and Ahmmad O I,'Design and implementation of robot control system for multistory buildings", Telekomnika vol.18, no .5. October 2020, pp. 2682 2689.

[7]. A. Vinodha Krishnan, J. Indira Priyadharshini and T. Sivaranjani, march 2017, "SMART home automation system using Arduino ",IJESRT ,Vol. 6, pp 170175. 